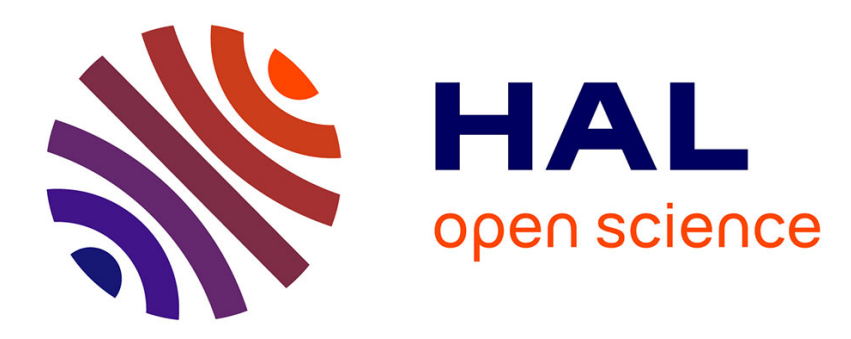

\title{
L'avenir des protectorats internationaux sur les Balkans
} Jacques Rupnik

\section{To cite this version:}

Jacques Rupnik. L'avenir des protectorats internationaux sur les Balkans: Présentation du Rapport de suivi de la Commission internationale indépendante sur le Kosovo. Critique Internationale, 2002, 16, pp.85-92. 10.3917/crii.016.0085 . hal-01017901

\section{HAL Id: hal-01017901 \\ https://hal-sciencespo.archives-ouvertes.fr/hal-01017901}

Submitted on 3 Jul 2014

HAL is a multi-disciplinary open access archive for the deposit and dissemination of scientific research documents, whether they are published or not. The documents may come from teaching and research institutions in France or abroad, or from public or private research centers.
L'archive ouverte pluridisciplinaire HAL, est destinée au dépôt et à la diffusion de documents scientifiques de niveau recherche, publiés ou non, émanant des établissements d'enseignement et de recherche français ou étrangers, des laboratoires publics ou privés. 


\section{D'ailleurs}

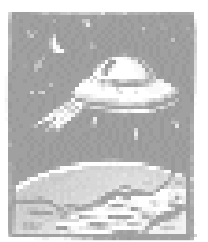

\section{L'avenir des protectorats internationaux sur les Balkans}

Présentation du Rapport de suivi de la Commission internationale indépendante sur le Kosovo

par Jacques Rupnik

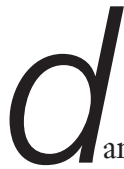

ans la décennie quatre-vingt-dix, la «communauté internationale » a eu pour objectif premier dans les Balkans de contenir, puis de clore la guerre de dissolution de la Yougoslavie : intervention et maintien de la paix étaient la préoccupation centrale. Celle de la décennie actuelle sera la prolifération des protectorats (ou semi-protectorats) internationaux dans la région, avec pour enjeu affiché la redéfinition du triptyque sécurité-stabilité-démocratie. Les leçons de la dernière décennie donnent clairement à voir que la prévention et la précocité de l'intervention sont essentielles. En Bosnie, où l'intervention s'est fait attendre, le bilan est de plus de 200000 morts ; au Kosovo, où elle a été rapide, il y en a eu environ 10000 ; en Macédoine, où l'engagement a été préventif, moins d'une centaine. Il en va de même pour les réfugiés et les personnes déplacées : trois millions en Bosnie, 900000 au Kosovo, quelques milliers en Macédoine. Par contre, l'après-intervention, la raison d'être et la mise en place des protectorats internationaux se définissent au cas par cas et dans l'improvisation. 
Au-delà de la présence militaire assurée par la SFOR en Bosnie et la KFOR au Kosovo, dont l'OTAN a été jusqu'à présent le coordonnateur, on assiste, en effet, à la mise en place d'une tutelle internationale en voie d'européanisation : Michael Steiner, le conseiller de Gerhard Schroeder pour la politique étrangère, a pris ses fonctions à la tête de la MINUK au Kosovo en février 2002, le Britannique Paddy Ashdown celle de « haut représentant » en Bosnie, tandis que le français Alain Le Roy, remplaçant François Léotard, gère à Skopje l'arbitrage du contentieux macédonien ${ }^{1}$. L'engagement international dans la région ne se limite pas à ces pays puisque tant la Serbie de l'après-Milosevic que, surtout, l'Albanie depuis 1997 connaissent aussi une forme de tutelle ou de supervision internationale. D'où une série de questions concernant ces protectorats. Comment est défini (ou comment peut-on redéfinir en cours de route) leur mandat? Sont-ils des solutions transitoires de courte durée vers une autonomie (self-government) annoncée ou bien présagentils, au contraire, une présence internationale de longue durée, moins soucieuse de préparer les transitions à la démocratie que de neutraliser les enjeux géopolitiques ?

Ce sont là certaines des questions qu'avait abordées le rapport de la Commission internationale sur le Kosovo, présidée par Richard Goldstone, ancien président du TPIY, présenté à Kofi Annan puis publié en octobre $2000^{2}$. Le texte dont la traduction est présentée ici est un « post-scriptum », rédigé un an après pour faire le point sur les changements intervenus depuis la chute de Milosevic et sur les résultats et les objectifs du protectorat international au Kosovo à la veille des premières élections législatives libres de novembre 2001.

La chute de Milosevic tourne incontestablement une page après une décennie guerrière, et l'on pourrait se contenter d'un tableau rassurant de l'alternance à Belgrade comme à Zagreb, du recul du nationalisme ethnique radical et de l'avènement de changements démocratiques favorisant, à terme, la coopération régionale. Le texte de la Commission ne manque pas de faire l'inventaire des changements positifs intervenus depuis l'intervention de l'OTAN au Kosovo au printemps 1999. Mais s'agit-il d'une paix imposée, ou d'une paix acceptée ouvrant une perspective de reconstruction et de réconciliation? Dans quelle mesure subsiste-t-il dans la région un potentiel de reprise ou de déplacement du conflit ? La thèse pessimiste veut qu'après une décennie dominée par la « question serbe » l'on soit désormais confronté (depuis l'intervention au Kosovo) à la « question albanaise ». C'est ainsi que fut souvent interprétée l'apparition d'une guérilla albanaise en Macédoine au printemps 2001 : forte de son succès au Kosovo, l'UCK (Armée de libération du Kosovo) aurait exporté sa stratégie de déstabilisation vers la Macédoine voisine (dont entre le quart et le tiers des habitants sont des Albanais) avec pour objectif ultime une « Grande Albanie », la réunion de tous les Albanais dans un même État. La Commission, sans ignorer les interactions entre le Kosovo et la Macédoine, suggère qu'il existait aussi d'autres causes liées tant aux faiblesses 
internes de l'État macédonien, peu enclin à mettre en œuvre une politique d'inclusion des albanophones dans ses institutions, qu'aux incertitudes concernant le statut final du Kosovo. Quels que soient les agissements et les arrière-pensées de certaines minorités radicales, poser ouvertement la question du statut final du Kosovo n'est peut-être pas le facteur déstabilisant pour la Macédoine ; bien au contraire, ce facteur pourrait consister à ne pas la poser, laissant ainsi à certaines mouvances nationalistes la possibilité de surenchères. Il est donc temps, comme le fait le document de la Commission, de se demander à quoi sert le protectorat.

L'objectif affiché, c'est d'abord la sécurité et la stabilité. Le moyen, à côté de la présence militaire internationale, serait de « gagner du temps » en évitant de poser la question du statut final du Kosovo. Lorsque, au lendemain d'une intervention militaire, l'on ne sait pas que faire d'un territoire, geler le statu quo passe pour la sagesse suprême. Chacun connaît les bonnes raisons de ne pas s'interroger sur le statut final : ne pas compromettre le fragile consensus au Conseil de sécurité de l'ONU, ne pas compliquer la tâche à la direction Kostunica à Belgrade, tenir compte des craintes ou de l'hostilité des pays voisins du Kosovo... Il existe pourtant au moins trois arguments pour aborder dès maintenant cette question controversée. D'abord, il est préférable de le faire dans un contexte relativement pacifié plutôt qu'à chaud, en période de crise. Ensuite, la résolution 1244 du 10 juin 1999 mettant un terme au conflit repose sur un postulat devenu problématique : le Kosovo fait partie de la Yougoslavie. Or c'est bien la question de l'après-Yougoslavie qui est posée depuis l'intervention de l'OTAN et la quasi défection du Monténégro, dont l'Union européenne a évité de justesse la déclaration d'indépendance en co-signant, avec son président et celui de la Yougoslavie, le 14 mars 2002 à Belgrade, un compromis négocié par Javier Solana qui reporte de trois ans

\footnotetext{
1. Aux « proconsuls » européens dans les Balkans il faut ajouter Erhard Busek, ancien ministre autrichien reponsable du Pacte de stabilité censé coordonner la reconstruction et la coopération régionales. Un début de coordination dans les priorités des protectorats européens commence à apparaître selon un axe double : d'une part, lutte contre le crime organisé, contrôle des frontières, lutte contre la corruption, le trafic d'armes et de stupéfiants, et, d'autre part, construction d'institutions démocratiques et de l'État de droit, «stratégie à long terme qui ramènerait les réformes économiques, judiciaires et militaires dans les Balkans sous le toit de l'UE », selon le compte-rendu d'une réunion de coordination publié dans le Financial Times du 13 mars 2002.

2. Independent International Commission on Kosovo, The Kosovo Report. Conflict, International Response, Lessons Learned, Oxford University Press, 2000. La Commission, créée en septembre 1999, était composée d'universitaires et de personnalités telles que Richard Goldstone, membre de la Cour constitutionnelle d'Afrique du Sud, Carl Tham, ancien ministre suédois de l'Éducation, ou Theo Sommer, directeur de l'hebdomadaire Die Zeit. La difficulté immédiate était d'écrire sur un sujet mouvant en évitant, autant que possible, de rester à la surface des problèmes et dans le diplomatiquement correct. C'est ainsi que le bilan et l'évaluation du « protectorat » sont liés à la question qu'occultent de préférence les représentants de la communauté internationale : celle du statut final du Kosovo. Les propositions qui prolongent l'analyse de la Commission n'ont d'autre but que d'attirer l'attention sur certaines implications ou effets pervers des options politiques concernant la présence internationale et le statut futur du Kosovo. C'est là, aussi, que se posent les questions de statut ou de déontologie entre « le chercheur et le commissaire ».
} 
le référendum sur l'indépendance. En attendant, la Yougoslavie est remplacée par la «Serbie-Monténégro », nouvelle entité hybride et provisoire. Cela donne en principe trois ans pour aborder le sujet qui fâche, le statut final du Kosovo ${ }^{3}$. Trois ans pour faire évoluer la position de Belgrade, des pays voisins et des « internationaux » chargés d'administrer le Kosovo. Mais comment préparer cette échéance si le sujet reste tabou? La troisième raison découle du divorce flagrant entre l'illusion entretenue par la résolution de l'ONU (le Kosovo fait partie de la Yougoslavie) et la réalité : le Kosovo est depuis trois ans complètement soustrait à l'influence militaire, administrative, économique de Belgrade. L'armée yougoslave a été remplacée par la KFOR, la monnaie est l'euro, un Parlement et un Président ont été élus avec l'objectif déclaré de mener le pays à l'indépendance. C'est ce divorce entre une fiction juridique et la réalité qui amène la Commission indépendante à confronter les protagonistes non pas à une solution toute faite (si elle existait, cela se saurait) mais aux conséquences des différentes options : protectorat international de durée indéfinie, partition, fédération yougoslave revue et corrigée, indépendance conditionnelle. Si la partition est à éviter (ne serait-ce que pour l'effet déstabilisateur qu'elle aurait sur le fragile édifice de Dayton en Bosnie-Herzégovine), on peut aussi imaginer une séquence entre ces options, où l'on passerait du protectorat international à une formule confédérale souple compatible avec un processus menant à une indépendance conditionnée par la communauté internationale.

Le second objectif proclamé du protectorat international au Kosovo est la mise en place d'institutions nouvelles et d'une transition vers la démocratie. Le problème prioritaire du Kosovo est effectivement l'établissement d'institutions, d'une administration, de services publics après le retrait de l'État serbo-yougoslave (dont les albanophones étaient de fait exclus depuis la suppression de l'autonomie du Kosovo par Milosevic). Ce fut sans doute la principale faiblesse de l'administration internationale, la MINUK dirigée à partir de juillet 1999 par Bernard Kouchner, que d'avoir sous-estimé l'aspect institutionnel alors que l'attention se focalisait soit sur les questions de sécurité soit sur l'assistance humanitaire au retour des réfugiés 4 . Ceci concerne la police et la justice et plus généralement l'incapacité d'agir dans la première phase, tant pour protéger la minorité serbe que pour combattre le développement de la criminalité organisée.

Veton Surroi, le directeur du principal quotidien de Pristina, Koha Ditore, décrit le problème comme suit : « L'apparition du crime organisé est d'abord liée à l'instauration de l'apartheid par Milosevic et son régime. Ensuite les guerres dans l'exFédération yougoslave ont apporté, au Kosovo aussi, la "culture de la criminalité" en commençant par la contrebande. Plus tard, l'insécurité et le flou général qui règnent toujours autour de la notion de propriété privée, apparus à la fin de la guerre au Kosovo, ont fourni les conditions idéales pour le développement du crime organisé. En l'absence d'ordre public, en l'absence de police et de tribunaux, le 
Kosovo est devenu un terrain idéal pour les crimes en tout genre $»^{5}$.

Autrement dit, la condition première pour mettre fin aux violations les plus flagrantes des droits de l'homme et instaurer les bases d'une transition démocratique est la création d'un État de droit. Mais comment construire des institutions d'État (administration, police, justice, etc.) si l'on ne sait pas quel État on construit? Le protectorat aura du mal à s'acquitter du premier aspect en occultant le second. Il en va de même pour les institutions démocratiques. Le « Représentant spécial » à la tête de la MINUK, Hans Haekkerup, a octroyé en mai 2001 un « cadre constitutionnel » au Kosovo ${ }^{6}$. Il permet l'élection d'un Parlement au suffrage universel direct, qui investit ensuite un Premier ministre et un Président. Les élections se sont déroulées dans des conditions jugées correctes par les «moniteurs » de l'OSCE qui, par ailleurs, gère aussi les inscriptions sur les listes électorales. Le nouveau Parlement et le gouvernement sont en place, mais à lire de près le cadre constitutionnel ils n'ont quasiment aucun pouvoir, à commencer par le seul qui vaille en démocratie : celui de contrôler le budget. Si le principe no taxation without representation (pas d'impôt sans représentation politique) est une base essentielle de la démocratie, le Kosovo n'en est certainement pas une. Il en va de même pour d'autres domaines cruciaux concernant l'avenir du pays, tels que les lois sur les médias ou sur les privatisations. Dans les deux cas, le Parlement kosovar est court-circuité et c'est la MINUK qui produit et promulgue la loi. D'où deux problèmes liés : d'abord le Parlement est une chambre où l'on parle, mais où l'on ne légifère pas sur les dossiers importants. Ainsi, en avril 2002, il débattait des questions de sécurité (les frontières, l'enclave de Mitrovica) et pas du dossier des privatisations. C'est le monde à l'envers : le Parlement débat de questions de sécurité qui théoriquement devraient rester entre les mains de la KFOR et de l'administration internationale, alors qu'il est écarté de la délibération sur les questions où il devrait

\footnotetext{
3. Voir International Crisis Group, Still Buying Time : Montenegro, Serbia and the European Union, Bruxelles, ICG, mai 2002. 4. L'approche de Bernard Kouchner fut tour à tour critiquée par Milosevic et Thaci (le leader de l'UCK), par les médias américains et par certains militaires français, ce qui donne à penser qu'il faisait du bon travail. Son bilan est aujourd'hui jugé très positivement par les Kosovars, surtout en comparaison de celui de son successeur, le Danois Hans Haekkerup, adepte d'une gestion jugée distante et autoritaire.

5. Veton Surroi, « Ne laissons pas notre pays aux criminels », en français dans Courrier International $n^{\circ} 599,25$ avril$1^{\mathrm{er}}$ mai 2002.

6. Pour sa version de « l'autonomie substantielle » accordée au Kosovo, voir Hans Haekkerup, « Waiting for Kosova selfgovernment » in Kosova \& Balkan Observer, janvier 2002, pp. 7-10. Le schéma institutionnel de l'administration internationale comporte quatre piliers coordonnés par le Représentant spécial du Secrétaire général de l'ONU : Affaires humanitaires (géré par le HCR), qui fut dissous en juillet 2000 étant donné la rapidité du retour des réfugiés ; Administration civile ; Construction de la démocratie : gouvernance et société civile (géré avec l'OSCE) ; Reconstruction (géré avec l'UE). Or le second pilier est composé de départements (agriculture, emploi, culture, environnement, administration locale, services publics, santé, justice, sports, jeunesse, transports) qui doublent de fait la structure du gouvernement. Sans faire des parallèles discutables, on ne peut s'empêcher de noter que, sous le communisme, les structures gouvernementales étaient aussi doublées de structures du parti et qu'aucun ministre ne prenait une décision importante sans le feu vert du département concerné du Comité central.
} 
faire son apprentissage de la démocratie. Mais surtout, une situation où un Parlement démocratiquement élu est systématiquement court-circuité par une administration internationale risque de déboucher rapidement sur un conflit de deux légitimités : celle de la communauté internationale qui a «sauvé » le Kosovo de la mainmise serbe, et celle des nouvelles instances kosovares démocratiquement élues.

La raison inavouée de ce verrouillage, c'est la crainte de voir le Parlement du Kosovo affirmer sa « souveraineté » et sa vocation à mener le pays à l'indépendance. Il est vrai que tous les leaders politiques sans exception se sont prononcés en faveur de celle-ci : le seul débat pour eux porte sur les moyens et les échéances. Résultat : lorsque, en novembre 2001, Hasim Thaci, l'ancien leader de l'UCK aujourd'hui à la tête de la deuxième formation politique du pays, a pris la parole à la première session du Parlement démocratiquement élu du Kosovo, le « Représentant spécial » lui a coupé le micro ${ }^{7}$ ! Or de deux choses l'une : soit le but du processus est de convertir d'anciens guérilleros en démocrates non violents, comme on le fait, ou cherche à le faire, en Irlande du Nord et au Timor (et il faut alors créer un espace politique pour gérer la question litigieuse), soit on considère par avance qu'évoquer les finalités du processus est une affaire trop sérieuse pour être laissée à l'incertitude du débat démocratique. Avec le risque de vider ce dernier de sa substance.

Pour l'instant, il s'agit d'une crise de légitimité latente étant donné la reconnaissance envers la communauté internationale, d'une part, et le caractère récent des nouvelles institutions, d'autre part. Une enquête sociologique récente, menée simultanément dans tous les pays des Balkans, révèle même une « exception kosovare $»^{8}$. Alors qu'à des degrés divers les pays de la région sont caractérisés aujourd'hui par une crise de confiance dans les institutions démocratiques et un pessimisme relatif quant à l'avenir, la population du Kosovo est résolument optimiste (les deux tiers répondent positivement à la question : «Votre pays évolue-t-il dans la bonne direction ? »9 ) et surtout révèle une confiance rare dans les nouvelles institutions démocratiques (Parlement, gouvernement, Président). L'optimisme s'explique en grande partie par le sentiment d'émancipation par rapport à ce que le Kosovo avait connu depuis la fin des années quatre-vingt sous Milosevic. La confiance dans les institutions repose plus sur une identification et une attente que sur un exercice réel du pouvoir. Ceci est confirmé par la confiance accordée à la police (75\%, contre $24 \%$ en Serbie) et à l'armée (83\%), puisque la police est récente et sa performance, pour le moment, peu convaincante, et que l'armée reste un objet virtuel dans un pays contrôlé par la KFOR sous commandement d'un général d'un pays de l'UE.

Cet « état de grâce » ne durera pas ${ }^{10}$. Une forte confiance dans des institutions nouvelles sans pouvoir réel risque de déboucher au Kosovo sur un phénomène déjà largement partagé dans les Balkans : la démocratie n'a pas de rival légitime, mais connaît déjà ses symptômes de crise et de défiance envers les institutions parlementaires ${ }^{11}$. Ceci est lié non seulement à la médiocre « performance » desdites institutions, mais 
aussi, comme le révèle l'enquête sociologique comparée, au divorce entre les priorités des élites et celles des populations ${ }^{12}$. Cette crise prématurée des institutions de la démocratie représentative (au sens où elle intervient avant même que la transition démocratique ne soit véritablement établie) est parfois perçue comme une justification du protectorat, alors que ce dernier contribue aussi à l'aggraver.

La plupart des États de la région sont des États en décomposition (failed states), c'est-à-dire qu'ils n'arrivent que partiellement à contrôler leur territoire et à fournir les garanties de sécurité tant au sens physique que social ${ }^{13}$. La présence internationale allant jusqu'au protectorat explicite se justifie par l'incapacité de l'État à garantir la protection élémentaire des droits à ses citoyens, laissant son territoire devenir une base de déstabilisation de ses voisins soit par intention (la Serbie et la « purification ethnique ») soit par « omission » (le Kosovo et l'absence d'institutions capables de s'opposer aux actes de violence contre la minorité serbe).

Cette symbiose entre des États en construction ou en reconstruction et la communauté internationale comporte aussi ses effets pervers pour la démocratie. En simplifiant beaucoup, les élites politiques dans les Balkans dépendent de trois assises : d'abord la communauté internationale, qui contribue à les financer et à les légitimer ; ensuite, les réseaux corrompus (ou mafieux), qui sont liés au pouvoir mais sont aussi intéressés par les dividendes de l'assistance internationale ; enfin seulement les

\footnotetext{
7. Le réflexe de couper le micro semble se répandre dans la gestion internationale des conflits balkaniques. C'est le juge du TPIY qui avait inauguré cette pratique en coupant le micro à Slobodan Milosevic lorsque celui-ci s'écartait des charges pesant sur lui pour dénoncer l'OTAN et le tribunal lui-même. Hans Haekkerup semble s'être érigé en juge et partie de la démocratie parlementaire au Kosovo.

8. Il s'agit de la première enquête de cette importance conduite simultanément dans une dizaine de pays des Balkans : le projet South East Europe Public Agenda Survey, lancé par International IDEA et réalisé par les principaux instituts dans les pays concernés capables de mener de telles enquêtes. Les premiers résultats ont été présentés à Pristina par J. Rupnik, conseiller du projet, à la réunion des ambassadeurs de l'OSCE dans les Balkans en avril 2002.

9. La Macédoine et la Bulgarie sont les plus pessimistes (moins de $20 \%$ croient à une évolution positive) alors que la Serbie se place derrière le Kosovo, avec environ $50 \%$.

10. Il est sans doute aussi lié à l'amélioration de la situation économique, à laquelle contribuent tant l'assistance internationale à la reconstruction que l'aide de la diaspora. Voir D.G. Demekas et al., Building Peace in South East Europe, Macroeconomic Policies and Structural Reforms since the Kosovo Conflict, Washington, Banque mondiale et Fonds monétaire international, 2002 ; D.G. Demekas, J. Herderschee, D. Jacobs, Kosovo, Institutions and Policies for Reconstruction and Growth, Washington, FMI, 2002.

11. Dans plupart des pays, on note une faible confiance dans les institutions politiques et dans le pouvoir, qui contraste avec la confiance envers des institutions symboliques telles que l'Église ou l'Université.

12. L'une des principales conclusions de l'enquête mentionnée est le décalage entre les priorités et les inquiétudes de la population, centrées sur les questions socio-économiques (chômage, pauvreté, corruption), et celles des élites du pouvoir, davantage préoccupées par la « question nationale».

13. Pour une perspective comparative sur cette problématique, voir M. Ignatieff, «Intervention and state failure », Dissent, hiver 2002, pp. 115-124. La question des protectorats internationaux abordée ici pour les Balkans se pose aussi, dans des contextes différents, en Afrique du Sud-Ouest ou en Afghanistan. La comparaison du Kosovo et du Timor oriental pourrait être intéressante, puisque c'est l'intervention au Kosovo qui a accéléré l'intervention au Timor, que le tout premier responsable de l'ONU au Kosovo en juin-juillet 1999 (S. Vieira de Melo) a été ensuite nommé au Timor où, à la suite d'élections générales, l'indépendance a été proclamée en mai 2002.
} 
électeurs, eux aussi intéressés par la présence internationale qui assure soit une aide humanitaire soit un contrepoids à l'imbrication du pouvoir politique et des réseaux corrompus ou mafieux ${ }^{14}$. Inutile de dire que, dans ces rent-seeking states, l'aide internationale, le clientélisme et la corruption vont souvent de pair. D'où l'importance de ne pas se payer de mots : qu'entend l'administration internationale par « autonomie substantielle », pour reprendre le langage de la résolution 1244 sur le Kosovo ? Quelle est la substance du self-government invoqué par le patron de la MINUK ? Le danger étant, c'est l'argument du texte ci-après, que le protectorat prolongé contribue à « infantiliser » une société où le pouvoir n'a déjà été que trop longtemps confisqué, et à restreindre l'espace démocratique. Le vrai but d'un protectorat devrait être d'œuvrer à sa propre dissolution. Pour ce faire, il faudra que les acteurs internationaux au Kosovo comprennent que le self-government, la responsabilité et la construction d'institutions étatiques n'a de chances de mobiliser les énergies, d'obtenir l'adhésion de la population aux institutions nouvelles que si l'on sait quel État on construit, que si la question de l'indépendance cesse d'être un tabou pour devenir, en toute clarté, une échéance qui se prépare. Il faudra parallèlement que les élites kosovares comprennent à leur tour que « l'indépendance » dont il est question n'est pas la souveraineté du $\mathrm{XIX}^{\mathrm{e}}$ siècle, mais une souveraineté partagée du XXIe siècle. Ils furent les bénéficiaires de l'engagement international face à un État qui confondait « souveraineté » et impunité pour ses violations massives des droits de ses citoyens ou pour son recours à la force. Ils devront faire l'apprentissage d'une souveraineté conditionnelle, c'est-à-dire qui se montre exigeante sur la renonciation à la violence ou le respect des minorités. Cette conception de la souveraineté partagée (et de l'indépendance conditionnelle) suppose surtout que le désengagement progressif des protectorats soit parallèlement accompagné par un processus d'intégration européenne. Le paradoxe actuel est que les pays qui ont le plus besoin de cette intégration dans l'Europe post-communiste sont précisément les moins prêts à l'assumer. La question pour l'après-Milosevic, au Kosovo comme dans le reste des Balkans, est bien celleci : comment passer du protectorat à l'intégration?

Jacques Rupnik est chercheur au CERI. Il a notamment dirigé Les Balkans. Paysage après la bataille, Bruxelles, Complexe, 1996. Il était membre de la Commission internationale indépendante sur le Kosovo qui a produit le rapport présenté ici. E-mail : rupnik@ceri-sciences-po.org

14. Il est intéressant de noter que, selon l'enquête Public Agenda Survey de janvier-février 2002, déjà mentionnée, c'est au Kosovo que la présence des institutions internationales est la mieux perçue (l'opinion la plus négative se rencontre en Serbie et en Republika Srpska). L'institution internationale la plus favorablement perçue est l'Union européenne : principal fournisseur d'aide et objectif politique à long terme. 


\title{
D'ailleurs
}

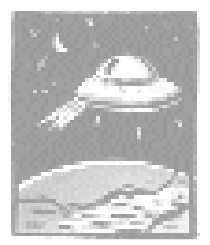

\section{L'indépendance conditionnelle : pourquoi ?}

\author{
Le rapport de suivi sur le Kosovo ${ }^{1}$
}

par la Commission internationale indépendante sur le Kosovo présidée par Richard Goldstone et Carl Tham

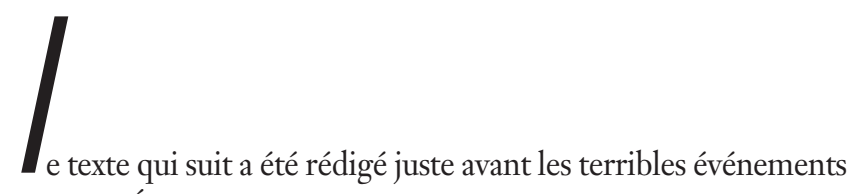
du 11 septembre 2001 aux États-Unis. La guerre contre le terrorisme à l'échelle mondiale a rejeté dans l'ombre presque toutes les autres questions internationales. Nous sommes très inquiets des conséquences que cela pourrait avoir pour la population du Kosovo. La guerre conduite par l'OTAN en 1999 s'achèverait en tragédie si le Kosovo était une fois de plus oublié ou, pire, si on laissait Belgrade libre de faire obstacle à son droit à l'autodétermination : certaines déclarations du gouvernement de la RFY ne sont pas rassurantes. La Commission considère de son devoir de rappeler aux dirigeants de la communauté internationale que le statut du Kosovo n'est toujours pas fixé, et que laisser cette question pendante est à la fois cruel pour les Kosovars et dangereux pour la stabilité des Balkans.

1. Ce texte est la traduction française (à l'exception de quelques annexes) de : Independent International Commission on Kosovo, The Follow-Up of the Kosovo Report. Why Conditional Independence?, Solna (Suède), 2001, 44 pages. 
La légitimité d'une intervention humanitaire ne dépend pas seulement de ce qui se passe pendant et immédiatement après celle-ci, mais aussi de son suivi à plus long terme. Dès lors qu'on s'engage dans une telle action, on a le devoir de veiller à ce qu'elle débouche sur des résultats positifs et stables.

Le rapport publié en octobre 2000 par la Commission internationale indépendante sur le Kosovo aboutissait à deux grandes conclusions. Premièrement, l'intervention de l'OTAN était illégale mais légitime. Illégale, parce qu'elle s'est faite sans l'assentiment préalable du Conseil de sécurité des Nations unies ; légitime, parce que de scandaleuses violations des droits de l'homme étaient en cours, que toutes les voies diplomatiques pour y mettre fin avaient été explorées en vain, et qu'elle a conduit à la libération du Kosovo, mis fin à l'oppression des Albanais de la province et permis le retour chez eux de tous ceux que les forces serbes avaient expulsés durant la guerre. Deuxièmement, il allait falloir accorder au Kosovo une « indépendance conditionnelle », c'est-à-dire qu'il lui faudrait un gouvernement autonome, en dehors de la République fédérale de Yougoslavie, mais dans un cadre international spécifique où la communauté internationale continuerait d'assurer la sécurité des frontières et la protection des minorités.

La publication du rapport a suscité un grand intérêt et force débats. La question posée le plus fréquemment aux membres de la Commission a été de savoir si ses propositions demeuraient d'actualité compte tenu des évolutions ultérieures, notamment de la chute de Milosevic et de l'avènement à Belgrade d'un nouveau gouvernement attaché à la démocratie, de l'extension de la violence à la Macédoine et au sud de la Serbie, enfin de la promulgation d'un nouveau cadre constitutionnel pour le Kosovo.

Selon certaines critiques, la persistance de la violence et d'un fort taux de criminalité à l'intérieur du Kosovo, les assassinats et les expulsions de Serbes et de membres d'autres minorités à titre de représailles, ainsi que la propagation de la violence dans les régions voisines remettent en question la légitimité de l'intervention de l'OTAN ; en outre, « l'indépendance conditionnelle » ne serait plus urgente, dans la mesure où la dictature brutale qui régnait à Belgrade a disparu ; pire, reprendre le débat sur le statut futur du Kosovo risquerait de perturber le fragile processus de démocratisation en Serbie. Il est indéniable que les événements récents ont inversé le sentiment majoritaire de par le monde, jusque-là plutôt positif envers les Albanais du Kosovo et négatif envers les Serbes.

La Commission a donc décidé de produire un additif à son rapport pour tenir compte de ces critiques. Il s'agit d'évaluer la pertinence de ses propositions initiales au regard de l'évolution de la situation dans les Balkans, de se demander si l'intervention de l'OTAN peut toujours être considérée comme légitime compte tenu des suites de l'intervention, et de vérifier que la proposition d'« indépendance conditionnelle » demeure valable. Nous commencerons par un tableau des évé- 
nements positifs et négatifs intervenus dans la région. Nous examinerons ensuite la situation au Kosovo même, en particulier les effets du nouveau cadre constitutionnel. Puis nous réexaminerons et préciserons la proposition d'«indépendance conditionnelle » et débattrons des mécanismes de mise en œuvre, et notamment de la nécessité d'un cadre régional.

\section{Évolutions intervenues dans les Balkans}

L'élément positif est que le nationalisme expansionniste et militariste n'est plus au pouvoir. Après une décennie au cours de laquelle la politique régionale a été dominée par des ethno-nationalistes radicaux, la chute de Milosevic a coïncidé avec l'arrivée au pouvoir, dans l'ensemble de la région, de non-nationalistes ou de nationalistes modérés favorables au changement démocratique et cherchant soutien et inspiration auprès de l'Occident, en particulier de l'Union européenne. À Belgrade, Vojislav Kostunica préside à une transition ambiguë : la démocratie, l'État de droit et l'indépendance des médias ont considérablement progressé, tandis que les structures et le personnel de l'appareil militaire et policier de l'ancien régime restent globalement inchangés. En outre, l'attachement au Kosovo en tant que symbole des aspirations nationales serbes est toujours présent au sein de la population et des pouvoirs publics. Le transfèrement de Milosevic à La Haye pour qu'il soit jugé par le Tribunal pénal international a surtout été dicté par des motivations économiques (afin de pouvoir bénéficier de l'aide de l'Occident) et a permis d'éviter un procès à l'intérieur du pays. Mais la découverte de charniers, qui en a été l'un des effets, a suscité en Serbie des interrogations sur le passé qui pourraient jouer un rôle important dans le processus de démocratisation, même si tout danger de réaction inverse n'est pas écarté. L'avenir constitutionnel de la Yougoslavie demeure incertain, notamment après l'élection au Monténégro - certes à une faible majorité - d'une coalition favorable à l'indépendance.

La Croatie a rompu encore plus nettement avec l'héritage de feu le président Franjo Tudjman, fait d'autoritarisme et d'extrémisme nationaliste. Le président Stipe Mesic s'est clairement engagé en faveur d'un renforcement de la coopération avec le Tribunal pénal international. L'arrestation du général Mirko Norac, en dépit de manifestations de grande ampleur à Split, a été un acte de courage politique que les capitales occidentales n'ont pas assez salué, de même que la rupture avec les ultranationalistes d'Herzégovine qui, soutenus à Zagreb par l'Union démocratique croate (HDZ), torpillaient depuis des années la mise en œuvre des accords de Dayton. Enfin, la Croatie s'est engagée à permettre le retour des Serbes expulsés de Krajina à l'été 1995.

La Bosnie-Herzégovine a, elle aussi, connu une évolution encourageante. Une coalition de partis non nationalistes et nationalistes modérés, l'Alliance pour le 
changement, est arrivée au pouvoir à la suite des élections de novembre 2000. Les sociaux-démocrates sont aujourd'hui la formation la plus importante de la Fédération croato-musulmane ; ils ont supplanté le Parti de l'action démocratique (SDA), parti nationaliste musulman de l'ancien président Alija Izetbegovic. Dans la Republika Srpska, le gouvernement de Milan Ivanovic semble favorable à la réforme démocratique, à la transparence et à la justice. Le retour des réfugiés s'est accéléré, même là où leur groupe ethnique n'est pas dominant. Toutefois, la volonté de coopérer avec le TPI, affirmée par les autorités, ne s'est pas encore concrétisée.

Il faut ajouter la modération constante de la Bulgarie, même si les conséquences de l'élection de l'ancien roi et celles de la guerre en Macédoine voisine demeurent encore imprévisibles. L'attitude de la Grèce dans l'ensemble de la région, en particulier en Macédoine, est de plus en plus constructive ; cette rupture tant attendue avec la politique d'agitation nationaliste des gouvernements précédents est due en grande partie à l'influence du ministre des Affaires étrangères Georges Papandréou. Par ailleurs, le gouvernement socialiste de l'Albanie s'est félicité des changements intervenus à Belgrade ; il a réaffirmé son soutien à l'intégrité des frontières et son opposition à l'emploi de la violence. Bref, le paysage offert par les Balkans semble plus prometteur qu'à l'époque où nous rédigions notre rapport : globalement, la région semble prête pour un changement démocratique, pour la stabilité et pour l'intégration régionales.

Cependant, si le projet nationaliste et expansionniste, cause des guerres dans les Balkans, paraît avoir été mis en échec au niveau gouvernemental, tous ces conflits ont laissé leurs traces dans les populations sous forme de sentiments extrémistes. Le traumatisme qu'ils constituent suscite des émotions violentes - peur, culpabilité, méfiance - difficiles à soulager et qui poussent à chercher le réconfort dans les certitudes illusoires de l'identification ethnique. En outre, la désintégration de la Yougoslavie et la guerre ont ruiné l'économie. Le PIB a très fortement chuté et le chômage connaît des taux sans précédent : 40 à 50 \% dans certaines zones. Une économie illégale liée aux réseaux criminels et aux groupes paramilitaires s'est mise en place dans toute la région au cours de la guerre, et beaucoup de gens dépendent pour vivre d'activités illégales, ou du moins informelles, « protégées » jusqu'ici par les partis nationalistes. Enfin, la guerre a affaibli la société civile et les forces politiques non nationalistes. Une certaine démocratie commençait à se développer en Yougoslavie dans les années 1990-1992, en particulier dans les villes. Mais ce sont précisément les citadins de niveau d'éducation élevé et peu marqués sur le plan religieux, socle d'une démocratisation potentielle, qui ont quitté le pays ou ont été tués ; cela est vrai en particulier en Bosnie-Herzégovine, en Croatie et en Serbie.

Cet héritage se traduit par l'audience persistante des partis nationalistes en certains points de la région. Les partis aujourd'hui au pouvoir à Belgrade ont large- 
ment axé leur campagne sur un programme nationaliste, quoique démocratique. En Croatie, le HDZ a obtenu de bons résultats aux dernières élections locales. En Bosnie-Herzégovine, malgré le succès des sociaux-démocrates, les observateurs extérieurs ont été déçus par les pourcentages de voix que continuent de recueillir les partis nationalistes. Au Monténégro, les partis favorables à l'indépendance ont obtenu une courte majorité aux élections d'avril 2001. En Macédoine, la victoire du parti nationaliste macédonien VMRO et du parti albanais ultra DPA en novembre 1998, après des années de gouvernement social-démocrate, a constitué un facteur important dans le déclenchement du conflit.

Plus important encore, les incidents, les mouvements de protestation et même la violence nationalistes persistent. On a mentionné plus haut les manifestations de Split. En Bosnie, il y a eu de violentes manifestations en Herzégovine occidentale contre les tentatives internationales de démantèlement des structures du pouvoir nationaliste, tout comme en Republika Srpska lorsque la communauté musulmane a voulu reconstruire des mosquées détruites pendant la guerre. De nombreux incidents ont également visé les réfugiés et les personnes déplacées rentrant chez elles, ainsi que la reconstruction de symboles culturels ou religieux.

Surtout, la violence dans le sud de la Serbie et en Macédoine souligne que la « question albanaise » est loin d'être réglée. Dans le sud de la Serbie, l'« Armée de libération de Presevo, Medvedja et Bujanovac » (UCPMB) a fait son apparition au début de l'année 2000. Ces trois districts avaient été ôtés au Kosovo après la Seconde Guerre mondiale, tandis que certaines zones de Serbie, pourtant majoritairement serbes, lui étaient au contraire ajoutées. Les raisons officielles invoquées avaient trait aux voies de communication vers la Grèce, l'Albanie et la Turquie, mais cela avait eu pour effet de réduire la proportion d'Albanais dans la population de la province. L'UCPMB s'est développée, ainsi que d'autres groupes armés, dans la « zone de sécurité terrestre » créée après l'arrivée des troupes de l'OTAN en juin 1999. Cette zone était supposée séparer les forces de l'OTAN des forces yougoslaves, et seuls des policiers serbes et monténégrins équipés d'armes légères étaient autorisés à y pénétrer. Après le changement politique à Belgrade, le nouveau gouvernement serbe a engagé des négociations avec l'UCPMB et l'OTAN, aboutissant à un accord qui concédait davantage de droits aux Albanais de la région, prévoyait la démobilisation, le désarmement et l'intégration des anciens combattants dans les forces de sécurité locales et autorisait les forces yougoslaves à revenir dans la zone de sécurité terrestre.

L'accord de Presevo constitue un véritable modèle pour ce type de négociations. La volonté d'aboutir du gouvernement serbe, les pressions exercées par l'OTAN sur la partie albanaise, l'implication à la fois des chefs des groupes armés et des Albanais de la région, ainsi que la confiance entre les diverses parties ont été les ingrédients du succès. La volonté tangible du gouvernement serbe de consulter 
directement le plus possible d'habitants de la zone a constitué une innovation déterminante qui pourrait beaucoup apporter aux efforts de paix dans d'autres régions du monde 2 . Reste à voir toutefois, à plus long terme, si l'accord sera effectivement appliqué.

La situation en Macédoine est très différente. L'Armée de libération nationale (ALN), tout comme l'UCPMB, s'est développée dans la zone de sécurité terrestre et, tout comme pour l'UCPMB, nombre de ses membres étaient d'anciens combattants de l'Armée de libération du Kosovo (UCK). En particulier, un grand nombre d'Albanais de Macédoine appartenaient au LPK (Levizja Popullarë e Kosovës), le parti politique de gauche qui avait initialement créé l'UCK et que finançait la diaspora par le biais du Fonds pour la patrie (Homeland Calling Fund). Comme pour l'UCPMB, on était en droit de se demander pourquoi des groupes d'activistes albanais avaient pu se développer à l'intérieur de la zone de sécurité terrestre et pourquoi la KFOR n'avait pas empêché leur approvisionnement. L'une des explications avancées est que cela était délibéré : avant la chute de Milosevic, les États-Unis voulaient déstabiliser le sud de la Serbie. C'est l'explication la plus en vogue dans les milieux tant serbes qu'albanais. Chez ces derniers, cela a contribué à développer le sentiment que les Américains étaient « de leur côté » et qu'il était possible d'agir en toute impunité. Une explication beaucoup plus réaliste est la réticence du commandement à faire courir des risques aux forces de maintien de la paix : la proportion des soldats de la KFOR ayant pour mission de protéger la force elle-même est telle que l'effectif restant est insuffisant pour assurer les missions de sécurité publique.

Diverses raisons ont été données à l'explosion de la violence en Macédoine en février 2001 : il s'agissait de protéger des itinéraires de contrebande de cigarettes ou une cache d'armes dont l'accès était interdit par un accord frontalier entre la Serbie et la Macédoine ; c'était une provocation d'anciens paramilitaires serbes dans la région ; ou encore, maintenant que le Kosovo était sur la voie d'une solution, il était temps de s'occuper du problème des droits des Albanais en Macédoine, et la preuve était faite que les méthodes violentes étaient plus efficaces que les voies pacifiques pour attirer l'attention. Quoi qu'il en soit, la violence s'est propagée beaucoup plus rapidement que prévu. L'actuel gouvernement est une coalition entre le VMRO et le DPA. Jusqu'aux élections de novembre 1998, les deux partis avaient adopté des positions extrêmes. Il arrivait au VMRO de se déclarer favorable à un État ethniquement homogène ; quant au DPA, il préconisait une fédéralisation de la Macédoine, avec une partie occidentale distincte, l'« Ilirida », sous contrôle albanais. Les deux partis ont mis en sourdine ce type de discours après avoir constitué un gouvernement de coalition. La situation en Macédoine n'avait rien à voir avec celle qui régnait au Kosovo sous la férule de Milosevic, avant l'intervention de l'OTAN. C'était une société démocratique, même si les Albanais, qui 
représentent un tiers de la population, avaient le sentiment d'être des citoyens de seconde zone. Et pas tout à fait à tort : la Constitution dispose en effet que la Macédoine est l'État du peuple macédonien (c'est-à-dire slave); la langue officielle est le macédonien et il n'y a pratiquement pas d'enseignement secondaire ou supérieur en langue albanaise ; le nombre des Albanais dans la fonction publique, en particulier dans l'armée et dans la police, est très faible 3 .

La situation en Macédoine ne ressemble pas non plus à celle qui règne dans la vallée de Presevo. Contrairement à la Serbie, le gouvernement macédonien, avec le soutien des gouvernements occidentaux, a riposté à la violence par une stratégie classique de contre-insurrection : bombardement des villages dans lesquels l'ALN était supposée se trouver, arrestation - et, semble-t-il, torture - de civils albanais afin d'identifier les membres de l'ALN ${ }^{4}$. Du fait de cette stratégie, la population albanaise, et même les partis qui avaient cherché (sans grand succès) à faire valoir ses revendications par des moyens pacifiques se sont ralliés à la cause de l'ALN. Les deux camps ont perpétré des déplacements de population : environ 120000 personnes ont dû quitter leurs foyers ; 80000 se sont réfugiées au Kosovo, dont 50000 sont à ce jour rentrées chez elles. Un accord de paix a été négocié en août 2001 et approuvé par le Parlement début septembre, sous la pression de la communauté internationale ; une force de l'OTAN a été envoyée en Macédoine pour collecter les armes remises volontairement par l'ALN. Cet accord répond à la plupart des revendications albanaises, mais la manière dont celles-ci ont été satisfaites augure mal de la confiance mutuelle et de la coopération entre les deux communautés à l'avenir. Les moyens violents mis en œuvre par la guérilla et par les forces gouvernementales ont sapé les bases de la confiance nécessaire à la viabilité d'un État fondé sur la coexistence de deux communautés.

Dans l'ensemble, nous ne sommes guère optimistes sur la maîtrise de la violence. Comme les dix dernières années nous l'ont appris, celle-ci est contagieuse dans la région. Elle se propage à cause de l'incertitude sur les frontières et sur le statut politique, au travers de réseaux criminels et paramilitaires ou par le biais des réfugiés

\footnotetext{
2. Des membres de la Commission ont rencontré Ivan Bender, le conseiller du vice-président Covic qui a négocié l'accord. C'était un nouveau venu au gouvernement, ancien militant étudiant qui avait soutenu les étudiants du Kosovo dans leur campagne pour l'application de l'accord sur l'éducation à l'automne 1997 (voir le chapitre I de notre rapport). Envoyé dans la région par Nebojsa Covic, il a passé plusieurs mois à visiter les villages, à discuter avec les habitants de leurs doléances et à gagner leur confiance, voire à les aider à s'occuper de leurs vaches. C'est certainement cette approche à partir de la base qui a donné à l'accord une fondation solide.

3. (Note de la rédaction) L'accord (mentionné quelques lignes plus bas dans le texte) intervenu en août 2001 sous l'égide de l'Union européenne entre les représentants des partis albanais et ceux des partis macédoniens slaves, et approuvé en novembre, après de nombreuses difficultés, par le Parlement, stipule que désormais des mesures concrètes seront prises pour favoriser une représentation plus équilibrée des Albanais dans l'administration en général et dans la police en particulier. Une université albanophone a également été créée.

4. Selon le rapport de Human Rights Watch sur la Macédoine, www.hrw.org
} 
et des déplacés, et elle est nourrie par les appétits tant des États voisins que de divers « entrepreneurs » de guerre ${ }^{5}$. Nombre des facteurs qui ont donné naissance à l'explosion de violence en Macédoine (fort taux de chômage, contrebande et autres activités illégales, incertitude, frustrations ethniques) existent ailleurs dans la région, par exemple au Monténégro.

\section{La situation au Kosovo et l'illusion de l'autonomie}

La situation au Kosovo est, elle aussi, caractérisée par des éléments positifs et négatifs. Les premières élections municipales libres se sont déroulées, de manière remarquablement pacifique, en octobre 2000. Les modérés de la Ligue démocratique du Kosovo (LDK), sous la direction d'Ibrahim Rugova, ont remporté une nette victoire : l'électorat a sanctionné les candidats issus de l'Armée de libération du Kosovo (UCK), menés par Hashim Thaci, car ces derniers restent associés à ses yeux à la violence et à la criminalité locales. Le nouveau Représentant spécial du Secrétaire général des Nations unies, Hans Haekkerup, a promulgué un cadre constitutionnel permettant la tenue d'élections générales en novembre 2001. Le nouveau gouvernement serbe coopère avec la MINUK, qui a ouvert un bureau à Belgrade. Un comité yougoslave de coopération avec le Kosovo a été créé à Pristina. Les nationalistes serbes les plus extrémistes sont ainsi marginalisés (par exemple, les financements destinés aux paramilitaires serbes à Mitrovica ont cessé) et les Serbes du Kosovo sont fortement encouragés à participer à la vie politique. Des forces de police internationales et locales ont été mises en place et un système judiciaire est en cours de création. La reprise économique est amorcée ; les recettes internes représentaient $75 \%$ du budget consolidé en 2001, et le soutien de la diaspora à la reconstruction s'est élevé au double des subventions publiques.

Du côté négatif, cependant, le niveau de criminalité et de violence reste inacceptable, malgré une certaine stabilisation. Si la violence ethnique a diminué, c'est surtout parce que les Serbes vivent désormais presque tous dans des enclaves et que leur liberté de circulation s'en trouve restreinte. Mitrovica est aujourd'hui divisée, tant économiquement que politiquement. La KFOR s'est montrée incapable et peu désireuse de mettre un terme à la violence persistante dirigée contre les minorités ethniques. C'est le plus grand échec depuis l'intervention de l'OTAN, et il faut le considérer comme une véritable tache morale, même si à l'évidence il était difficile à éviter. Il subsiste des explosions de protestation et de violence nationalistes, particulièrement à Mitrovica où des « guetteurs » postés sur la partie serbe du pont veillent à préserver la division de la ville. Les dirigeants politiques albanais du Kosovo se déclarent certes hostiles à la violence mais ne font aucun effort réel pour s'en rendre maîtres, par exemple en exerçant des pressions sur les militants de base, en engageant un dialogue et une coopération véritables avec les Serbes ou, 
plus généralement, en s'efforçant de créer une atmosphère différente ; surtout, ils gardent une attitude équivoque sur les événements de Macédoine et du sud de la Serbie, en évitant de condamner ouvertement l'UCPMB ou l'ALN. Enfin, le taux de chômage officiel reste élevé, de l'ordre de 50 \%, ainsi que l'activité économique illégale - construction sauvage, racket, contrebande ou trafic. Ces activités découragent l'investissement intérieur et étranger.

Dès les débuts de la MINUK et de la KFOR en juin 1999, l'incertitude quant au statut final du Kosovo a créé des tensions entre les acteurs de la vie politique locale et les représentants de la communauté internationale ainsi qu'au sein de cette dernière, comme nous l'avons souligné dans notre rapport. La résolution 1244 de l'ONU stipulait que la MINUK devait préparer le Kosovo à une autonomie et à un autogouvernement substantiels au sein de la RFY. Elle envisageait même de maintenir une présence de la police de la RFY au Kosovo. Toutefois, après ce que la population du Kosovo avait subi de la part des autorités serbes et yougoslaves (négation des droits civils, attaques contre les villages, massacres, et finalement expulsions généralisées), tout exercice de la souveraineté de la RFY sur le Kosovo était, par simple réalisme, à exclure dès juin 1999. De même, « l'autonomie et l'autogouvernement substantiels » n'étaient acceptables par la population albanaise que comme étape vers une forme d'indépendance. L'administration de la MINUK, et plus généralement la communauté internationale, étaient divisées entre ceux qui considéraient l'administration de l'ONU comme un instrument transitoire destiné à préparer les habitants du Kosovo à l'autonomie et ceux qui y voyaient un protectorat durable. Lors de ses nombreuses visites au Kosovo, la Commission a été frappée par la condescendance très impériale de certains éléments de l'administration internationale à propos de la capacité des habitants du Kosovo à s'autogouverner.

Le Cadre constitutionnel d'autogouvernement provisoire promulgué en mai 2001 par le Représentant spécial du Secrétaire général des Nations unies, Hans Haekkerup, après des consultations restreintes avec les acteurs de la vie politique locale 6 , a fixé les règles du jeu pour la période précédant et suivant les élections générales de novembre 2001. Il prévoit une Assemblée avec une garantie de représentation

\footnotetext{
5. Voir : European Stability Initiative on Montenegro, « Rhetoric and reform : A case study on institution building in Montenegro, 1998-2001 », Podgorica et Berlin, 1er juillet 2001, www.esiweb.org

6. Mme Flaka Surroi, qui représentait la société civile dans la commission chargée d'élaborer le Cadre constitutionnel, a démissionné en invoquant les raisons suivantes : « Premièrement, le Cadre constitutionnel a été adopté sans même que l'on ait demandé leur avis aux habitants du Kosovo. Il a été élaboré par un groupe d'experts et discuté uniquement avec les partis politiques. Inutile de préciser que la société civile n'a pas du tout été consultée. Deuxièmement, selon les dispositions de ce document, le Kosovo élira des structures d'autogouvernement dotées d'un pouvoir extrêmement limité. Enfin, je ne saurais faire partie de l'équipe qui devra fixer les règles d'élections destinées à mettre en place un système concentrant la quasitotalité des pouvoirs entre les mains d'une personne et de ses assistants, les règles d'élection d'une Assemblée législative dont chaque décision pourra se heurter à un veto, et les modalités de désignation d'un Président dont le rôle se bornera à décerner des récompenses et à exprimer des remerciements ».
} 
pour les minorités ethniques, un Président élu par cette assemblée et un Premier ministre et un gouvernement choisis par le Président. Le gouvernement du Kosovo peut gérer ses affaires intérieures (économie, éducation, transports, administration locale, pouvoir judiciaire, prisons et médias), mais ce pouvoir est limité par toute une série d'attributions réservées au Représentant spécial : celui-ci peut dissoudre l'Assemblée et convoquer de nouvelles élections, fixer « les paramètres financiers et les grandes orientations » du budget, nommer et révoquer les juges et les procureurs, contrôler la Force de protection du Kosovo, les douanes, la direction du logement et des biens immobiliers, désigner tous les hauts responsables économiques, surveiller l'ensemble des relations extérieures et assurer la coordination avec la KFOR en matière de sécurité intérieure et aux frontières.

Les larges pouvoirs accordés au Représentant spécial signifient qu'au lieu de l'autogouvernement substantiel promis aux habitants du Kosovo par la résolution 1244, ceux-ci n'obtiendront en fait qu'une autonomie très limitée, et pour tout dire illusoire. Ces dispositions constitutionnelles apparaissent dictées par une méfiance généralisée à l'égard des capacités administratives et politiques de la population. Or si l'on se défie de la population, celle-ci, selon toute probabilité, se montrera méfiante à son tour. La Commission considère que les habitants ne se comporteront pas de manière responsable tant qu'on ne leur aura pas confié de responsabilités. Il y a lieu de craindre qu'une fois désignés le Président, le Premier ministre et le gouvernement du Kosovo, à la suite des élections de novembre 2001, tout sera en place pour des litiges toujours plus nombreux autour des pouvoirs réservés du Représentant spécial, et plus généralement entre une élite politique locale confirmée par l'électorat, désireuse d'obtenir plus de pouvoir dans le cadre de l'autogouvernement, et une administration internationale cherchant à préserver ses prérogatives. Le conflit qui couve depuis longtemps entre les conceptions internationales de l'autogouvernement et celles des habitants pourrait alors évoluer vers une crise constitutionnelle, qui ferait obstacle au développement économique et social à long terme.

Cette situation mal résolue a aussi des répercussions extérieures. La « question albanaise » est liée au fait que la majorité de la population albanaise s'est trouvée, après la Seconde Guerre mondiale, en dehors de l'Albanie, principalement dans ce qui était alors la Yougoslavie. Aujourd'hui, il y a quelque 400000 Albanais en Serbie, près de 2 millions (90\% de la population) au Kosovo, 600000 à 700000 en Macédoine sur une population totale de 2 millions d'habitants, et 40000 au Monténégro. En Albanie même, 95 \% des 3,5 millions d'habitants sont albanais. Si certains ont sans doute rêvé d'une Grande Albanie durant l'ère communiste, ce rêve a volé en éclats après le contact avec la réalité, en particulier après la guerre de 1999, lorsque de nombreux Albanais du Kosovo se sont trouvés réfugiés en Albanie jusqu'à la fin du conflit. L'expérience relativement libérale des Albanais d'ex- 
Yougoslavie, qui avaient souvent travaillé en Europe occidentale, est radicalement différente de celle des Albanais d'Albanie, qui vivaient dans la société la plus staliniste-maoïste et la plus fermée d'Europe. Nombreux sont les Serbes et les Macédoniens qui croient que l'objectif des Albanais est la Grande Albanie, mais la plupart de ces derniers, y compris les combattants de la guérilla, affirment qu'ils ne veulent que l'indépendance du Kosovo, la garantie des droits des Albanais en Serbie, en Macédoine et au Monténégro, ainsi que des frontières suffisamment ouvertes pour que les Albanais puissent circuler librement et rencontrer leurs proches établis dans différents pays. Sur ce dernier point, ils citent souvent l'exemple de l'Allemagne, de la Suisse et de l'Autriche, où bon nombre d'entre eux ont vécu.

L'incertitude quant au statut futur du Kosovo a pour effet que la question albanaise n'est pas réglée et que, par conséquent, celle des frontières reste en suspens. Ce n'est pas, comme le craignent certains, l'indépendance du Kosovo qui pourrait avoir un effet dominos en encourageant les revendications d'autonomie territoriale ailleurs, mais bien plutôt l'incertitude sur l'avenir qui donne à penser à certains acteurs que tout reste à jouer et que le sud de la Serbie et certaines parties de la Macédoine peuvent encore entrer dans l'équation. En d'autres termes, les conflits récents peuvent être considérés comme un effet de l'incertitude, celle-ci incitant les acteurs à essayer de changer les choses sur le terrain avant qu'un règlement définitif ne soit trouvé. Incertitude et instabilité agissent comme des « mécanismes de contagion $\gg$.

\section{Réexamen de l'idée d'indépendance conditionnelle}

La résolution 1244 du Conseil de sécurité des Nations unies n'a pas défini l'avenir à long terme du Kosovo. La proposition d'indépendance conditionnelle repose sur un fondement normatif : elle découle des agressions systématiques perpétrées pendant des années à l'encontre des droits des Albanais du Kosovo et de la conséquence de ces agressions, le rejet par ces derniers de l'autorité serbe. Dans son rapport, la Commission examinait concurremment quatre autres options : partage, protectorat de durée indéterminée, indépendance pleine et entière, autonomie au sein d'une République fédérale de Yougoslavie démocratique. Certains estiment que l'évolution intérieure et extérieure décrite dans les précédentes sections rend telle ou telle de ces autres options plus souhaitable. Nous pensons toutefois que ces nouveaux éléments ne modifient pas le fondement normatif de l'indépendance conditionnelle. De plus, et indépendamment du fait que le contexte est encore susceptible de se modifier, la Commission est parvenue à la conclusion que les raisons pour lesquelles elle avait rejeté les quatre autres options demeurent valables, et même sont encore renforcées par les derniers événements. Résumons brièvement ces motifs : 
Le partage. Cette éventualité a récemment été remise en avant, en particulier par le vice-président serbe Covic, qui a laissé entendre que le Kosovo pourrait être divisé, sa partie Nord devenant une entité analogue à la Republika Srpska. Dans notre rapport, nous insistons sur une objection de principe au partage : il enfreindrait «l'engagement moral pris par la communauté internationale d'éviter les déplacements de populations et de soutenir le caractère multi-ethnique et mixte des sociétés balkaniques » (p. 267). Mais il existe également des objections d'ordre pratique : un partage ne serait jamais accepté par la majorité albanaise qui, à tort ou à raison, considère que le complexe minier de Trepca, dans le nord du Kosovo, constitue l'un des principaux actifs de ce dernier. Cette option risquerait fort de déboucher sur un regain de violence dans la vallée de Presevo, dans le but d'échanger Mitrovica contre Presevo.

Le protectorat de durée indéterminée. C'est l'option qui découle du nouveau Cadre constitutionnel. Elle est inacceptable pour toutes les raisons mentionnées cidessus. Même si elle est susceptible d'atténuer les incertitudes dans le cas où l'indépendance serait clairement exclue, la tension entre l'administration internationale et les revendications d'autodétermination des Kosovars risquerait d'atteindre un point de rupture.

L'indépendance pleine et entière. Tant que les violences politiques continueront, aussi bien au Kosovo que dans les régions voisines, la perspective d'indépendance totale est exclue. Pour dire les choses en termes simples, une indépendance totale serait inacceptable, dans le contexte actuel, aussi bien pour la minorité de la population que pour les États voisins, et n'aurait que peu de chances d'être approuvée par le Conseil de sécurité des Nations unies, de l'autorité duquel dépend le statut ambigu actuel.

L'autonomie au sein d'une RFY démocratique. Le renversement de Milosevic rend effectivement cette quatrième option plus attrayante, tant pour Belgrade que pour la communauté internationale. Une idée souvent avancée est celle d'une organisation en « trois républiques » dans laquelle le Kosovo deviendrait, au sein d'une Yougoslavie reconstituée, une république ayant le même statut que la Serbie et le Monténégro ; la résolution 1244 se réfère à la souveraineté de la Yougoslavie, non à celle de la Serbie, ce qui ouvre la possibilité de séparer le Kosovo de la Serbie mais non de la Yougoslavie. Il va sans dire que cette option dépend de ce qui se passera au Monténégro. À la suite de la courte victoire des partis monténégrins indépendantistes aux élections d'avril 2001, il existe une possibilité réelle d'indépendance de cette république, auquel cas la question de l'indépendance du Kosovo se posera avec encore plus d'acuité. Il se peut également que, du fait que la société monténégrine est profondément divisée sur la question de l'indépendance, un compromis temporaire soit trouvé avec la Serbie dans le sens d'une confédération lâche, au moins pour un temps. Quoi qu'il en soit, il devra y avoir une coopération aussi bien dans 
le cadre de la Yougoslavie qu'entre Pristina et Belgrade. Mais la question du statut futur des Kosovars ne doit pas dépendre de l'évolution du reste de la Yougoslavie. C'est là une des leçons à tirer des événements de la décennie écoulée : on avait demandé aux Albanais du Kosovo de suspendre leurs revendications pendant que l'on traitait les problèmes de la Croatie et de la Bosnie, ce qui n'a fait qu'alimenter leurs griefs. Dans son rapport, la Commission faisait valoir que, même en cas de démocratisation des autres parties de la Yougoslavie, il faudrait malgré tout rejeter cette option car, après tout ce que les Albanais du Kosovo avaient subi de la part des autorités de la RFY durant plus de dix ans, il serait déraisonnable d'attendre d'eux qu'ils se soumettent à une quelconque souveraineté, même purement formelle, de cette dernière. Cet argument demeure valable. De fait, maintenant que le Kosovo échappe en pratique à l'autorité du système administratif et juridique serbe, il est encore plus irréaliste d'imaginer que les Albanais du Kosovo puissent être à nouveau disposés à accepter la présence de fonctionnaires représentant la Serbie, à payer des impôts à Belgrade ou à solliciter la délivrance de passeports auprès d'un État qui a expulsé la moitié d'entre eux et détruit leurs pièces d'identité pour s'assurer que tout lien avec lui était définitivement rompu. Même si, pour le moment au moins, la RFY est dirigée par des hommes nouveaux.

L'indépendance conditionnelle est donc l'option qui garde la faveur de la Commission. Comme certains acteurs de la communauté internationale paraissent estimer que l'autogouvernement prévu par les principes constitutionnels de mai 2001 équivaut à une indépendance conditionnelle, de fait sinon dans les termes, il importe d'expliciter la différence entre les deux. Dans l'autogouvernement sous l'autorité de la MINUK, l'administration des Nations unies conserve un large droit de regard sur la politique intérieure, la police et la justice, ainsi que sur l'ensemble de ce qui relève des relations internationales. Ces pouvoirs s'apparentent à ceux d'un vice-roi et sont plus proches d'une situation de dépendance coloniale que de celle d'un peuple disposant de lui-même. Dans la notion d'indépendance conditionnelle que défend la Commission, les attributions d'ordre interne actuellement réservées au Représentant spécial seraient progressivement transférées au gouvernement du Kosovo ; à la fin du processus, le Kosovo serait indépendant - quel que soit le caractère conditionnel de cette indépendance - hors de la RFY. On ne voit aucune raison cohérente qui justifierait que les Kosovars n'aient aucune prise sur « les paramètres financiers et les grandes orientations » de leur propre budget, surtout si l'on tient compte du fait que plus de $70 \%$ des recettes de la province proviennent de sources internes et non internationales. De même, la Commission ne voit aucun motif convaincant pour justifier l'idée que le gouvernement du Kosovo n'ait aucune autorité sur des domaines comme les douanes, la justice, la police, la propriété publique, étatique et sociale, les chemins de fer et les transports, l'aviation civile, la direction du logement et des biens immobiliers, la 
propriété commerciale, le tracé des limites des communes, la réglementation applicable aux armes à feu, en un mot sur l'ensemble des pouvoirs réservés au Représentant spécial en vertu des chapitres 8.1, alinéas $a$ à $z$, et 8.2, sans parler des pouvoirs résiduels non définis attribués au Représentant spécial en vertu du chapitre 12. Et, s'il n'existe aucune bonne raison pour laquelle ces pouvoirs devraient échapper au gouvernement du Kosovo, on peut être sûr qu'une lutte se développera autour de ces enjeux.

Outre les pouvoirs d'ordre interne mentionnés ci-dessus, la Commission estime que le gouvernement du Kosovo devrait avoir celui de négocier avec la force internationale de sécurité, la KFOR, et de lui faire des représentations, ainsi que d'engager des négociations avec les organisations internationales et les gouvernements étrangers. Il importe tout particulièrement que le nouveau gouvernement du Kosovo établisse des relations pacifiques avec l'ensemble de ses voisins, surtout avec la Macédoine et la Serbie. Si les relations internationales de la nouvelle entité restent uniquement aux mains du Représentant spécial, rien n'incitera les Kosovars à un comportement responsable à l'égard de leur entourage.

Quelles seraient, dans le cas envisagé, les limites imposées à l'autonomie ? La nature exacte des conditions dont l'indépendance sera assortie ne pourra que résulter d'un processus de délibération, à la fois à l'intérieur du Kosovo et avec ses voisins. En guise de point de départ de la réflexion, la Commission propose les quatre conditions que voici :

Renoncer expressément à toute modification des frontières, c'est-à-dire à tout projet de Grande Albanie, voire de Grand Kosovo (qui comprendrait, par exemple, la vallée de Presevo ou l'ouest de la Macédoine).

Cela signifie que les responsables politiques kosovars doivent rejeter la logique nationaliste selon laquelle les frontières doivent coïncider avec la répartition des groupes ethniques. Ils pourront soutenir les droits des Albanais dans les autres pays et la coopération avec leurs voisins, mais devront énoncer clairement leur engagement en faveur de la non-ingérence et de l'intégrité territoriale.

Garantir, dans un cadre constitutionnel, les droits de l'homme pour tous les citoyens du Kosovo.

Les minorités devront jouir de l'égalité d'accès et de participation aux institutions du Kosovo, y compris la justice, la police et les fonctions électives ; leur droit à des services publics et à un enseignement dans leur langue sera placé sous protection internationale. Ces garanties constitutionnelles devront être bien davantage qu'un engagement sur le papier : elles devront être intégrées à la vie politique du Kosovo en termes de modes de pensée et de comportements. Il devra donc y avoir de réels efforts de coopération, de dialogue et de réconciliation entre les différents groupes. Il faudra en particulier viser à établir les conditions qui permettront aux Serbes ayant 
quitté la province d'y revenir s'ils le souhaitent, et à permettre aux minorités qui y sont restées de prendre part à la vie sociale.

Renoncer à l'usage de la violence pour régler les différends intérieurs ou extérieurs.

Les responsables politiques comme la société civile devront déployer des efforts réels pour instaurer au Kosovo une culture de la non-violence. Il ne suffira pas d'y prêter verbalement allégeance. Il faudra des mesures significatives et visibles de condamnation et de prévention, à l'intérieur comme à l'extérieur. Les responsables politiques devront renoncer clairement à toute tentative de recours à la force à l'appui des revendications des Albanais ; ils devront également se démarquer clairement de groupes tels que l'UCPMB ou l'ALN.

S'engager en faveur de la coopération régionale, de la gestion des affaires publiques au niveau régional et d'institutions régionales durables.

La supervision effective de ces conditions exige une présence internationale durable, chargée particulièrement de la protection des frontières et des minorités. Le Kosovo étant une société profondément divisée, il aura encore longtemps besoin non seulement d'une présence internationale de sécurité, mais aussi de l'aide de la communauté internationale. Il faudra mettre en place un mécanisme institutionnel permettant à celle-ci d'intervenir en cas de violences intercommunautaires ou d'atteintes aux droits des minorités qui menaceraient la stabilité de la province lorsque le gouvernement du Kosovo se révélera incapable ou peu désireux d'agir, ou encore pour prévenir toute ingérence armée dans les États voisins. Les pouvoirs de la communauté internationale, qui pourront ou non continuer à être confiés au Représentant spécial, devront donc être limités à la protection des minorités, à la garantie des droits de la personne et à celle de l'intégrité des frontières ; ils ne devront s'exercer que lorsque les autorités locales élues ne seront manifestement pas en mesure de s'acquitter des obligations qui leur incombent en vertu de la Constitution. À plus longue échéance, à mesure que le gouvernement du Kosovo aura mis en place ses capacités administratives et politiques et que la situation de la région sera devenue plus favorable à la paix, il devrait être possible de réduire encore le rôle de la communauté internationale et de confier au peuple du Kosovo des responsabilités plus étendues. Tel est l'avenir contenu dans l'idée d'indépendance conditionnelle. Il se distingue nettement de l'autonomie limitée sous l'autorité de la MINUK.

L'indépendance conditionnelle constitue à la fois un but et un processus. Le but est celui de la souveraineté et de l'indépendance du peuple du Kosovo dans les limites définies par la communauté internationale, à condition que celui-ci respecte l'intégrité territoriale de ses voisins, assure à l'intérieur la paix et le respect des droits de l'homme, et protège les traditions et les institutions de la communauté minoritaire. L'indépendance conditionnelle n'exclut pas (elle l'appelle très probablement, 
au contraire) le développement de relations étroites avec les voisins, notamment avec l'entité, quelle qu'elle soit, qui remplacera la RFY. Mais la meilleure base pour de telles relations sera celle d'un mouvement volontaire, à partir d'une indépendance souveraine ; et il revient au peuple du Kosovo, dans le cadre de libres négociations avec ses voisins, de définir ce qu'elles devront être. L'indépendance conditionnelle est également un processus graduel de dévolution dans le cadre duquel les attributions actuellement exercées par la communauté internationale, en la personne du Représentant spécial, seront peu à peu transférées à des responsables locaux élus. Elle n'a pas pour but de conserver à la communauté internationale les prérogatives de la responsabilité politique, mais au contraire de restituer celle-ci à ceux qui doivent en être les détenteurs, les habitants du Kosovo.

Trois arguments principaux sont avancés à l'encontre de cette proposition. Le premier a trait à la démocratie en Serbie. Aussi bien chez les responsables serbes que parmi les commentateurs internationaux, on fait souvent valoir que le moment est mal venu pour poser la question du statut futur du Kosovo car cela pourrait entraver la marche de la Serbie vers la démocratie, le sujet étant susceptible d'être exploité par des extrémistes, ou simplement pouvant détourner l'attention de tâches plus urgentes. Il ne fait pas de doute que l'indépendance conditionnelle constitue, pour beaucoup de Serbes, une pilule bien difficile à avaler. Il n'en est que plus important de faire en sorte que les responsables albanais du Kosovo entrent au plus tôt en rapport avec Belgrade et engagent la discussion sur les modalités d'établissement de relations nouvelles. Celle-ci devra porter sur la mise en place des conditions permettant le retour des réfugiés, sur la préservation des sites historiques serbes dans la province, sur la recherche des personnes disparues et les échanges de prisonniers, ainsi que sur la coopération économique.

Il y a encore 3000 à 4000 personnes disparues du fait de la guerre, pour la plupart Albanais du Kosovo. Il est légitime que tant les Serbes que les Albanais du Kosovo demandent à la MINUK et aux autres autorités compétentes de mettre en œuvre plus de moyens et d'énergie pour découvrir ce qu'il est advenu d'elles. La plupart sont sans doute mortes. Et, jusqu'ici, les exhumations au Kosovo et en Serbie ont été beaucoup trop lentes.

200 à 300 Albanais du Kosovo sont encore détenus comme prisonniers politiques en Serbie. Le gouvernement de Belgrade doit les relâcher dès que possible. C'est un pas important sur la voie de la réconciliation.

La Commission estime que, si cette question est reportée à plus tard, elle sera probablement plus difficile à traiter, cela non seulement au Kosovo, comme nous l'avons vu, mais encore en Serbie même. De même que dans le cas de la coopération avec le TPI, il est plus commode de prendre des décisions difficiles dès à présent, alors que le régime qui a succédé à Milosevic jouit d'une grande popularité, que plus tard, quand l'euphorie se sera dissipée. L'indépendance conditionnelle du 
Kosovo pourrait être perçue comme le prix à payer pour libérer la démocratie serbe des illusions nationalistes, ouvrir la voie à une réconciliation entre la Serbie et ses voisins et offrir la perspective d'un « retour vers l'Europe ». Après dix années de guerre, la société serbe est épuisée et aspire à une vie « normale ». Kostunica pourrait faire bon usage de son brevet de nationalisme et de son mandat démocratique en rompant clairement avec l'ère Milosevic et avec le mythe de la « reconquête du Kosovo ». On a souvent tendance en Serbie à considérer le 5 octobre 2000 comme le premier jour d'une ère nouvelle ; mais la Serbie doit tout de même régler ses comptes avec son passé, à la fois pour retrouver une situation « normale », se démocratiser et développer la coopération régionale.

Le deuxième argument que l'on oppose à l'indépendance conditionnelle est celui des dominos : elle risquerait d'encourager les revendications d'indépendance du Monténégro et de partage de la Bosnie ou de la Macédoine, sans parler de celles qui s'expriment en Serbie même, en Voïvodine, par exemple, ou encore dans le Sandjak. Cet argument peut être rejeté pour quatre raisons. Primo, les conditions imposées à l'indépendance excluent toute contagion directe à partir du Kosovo : la renonciation à la logique nationaliste, le refus de la violence et l'engagement en faveur de la non-ingérence rendent impossibles des effets directs sur les entités voisines ; et le maintien de la présence internationale garantit ces conditions. Secundo, l'indépendance du Kosovo supprimera l'incertitude résultant du caractère provisoire de son statut actuel, d'où une moindre incitation à modifier la situation sur le terrain, comme nous l'avons vu. Tertio, rappelons que le choix de l'indépendance du Kosovo repose sur une base normative. En termes juridiques, ce qui plaide en faveur de l'autodétermination des Albanais du Kosovo, ce sont les atteintes systématiques aux droits de l'homme qui se sont perpétrées pendant si longtemps. Ni les Serbes de Bosnie ni les Albanais de Macédoine ne peuvent se prévaloir d'un vécu analogue. Bien au contraire, il conviendra de démythifier résolument les prétentions de tout groupe qui, comme en Macédoine par exemple, ose affirmer que sa situation est comparable à celle que les Albanais du Kosovo ont connue jusqu'en 1999. Quarto, enfin, on voit difficilement en quoi l'une ou l'autre des autres options avancées serait moins susceptible d'avoir des effets de type dominos. Les responsables politiques du Kosovo doivent très vite, comme leurs homologues serbes, engager le dialogue avec leurs voisins afin de les rassurer sur leurs intentions, notamment en matière de respect de l'intégrité territoriale.

Le troisième argument avancé contre tout changement de statut du Kosovo concerne le Conseil de sécurité. On fait valoir que toute tentative d'aller au-delà de la résolution 1244 remettrait en question le compromis avec la Russie - en vertu duquel le Kosovo devra rester au sein de la Yougoslavie - qui a permis de mettre fin à l'intervention militaire de l'OTAN. Il n'en reste pas moins, comme nous l'avons mentionné, que le statu quo est intenable. Certes, la Russie émettrait des 
objections à l'indépendance du Kosovo, mais il est inconcevable qu'elle aille entraver une solution dès lors qu'un accord aura été trouvé sur place. Il n'en est que plus important pour l'OTAN et pour l'Union européenne d'exercer une pression sur les gouvernements de la région pour parvenir à un tel accord, fondé sur la réalité d'aujourd'hui.

La souveraineté était conçue, au XIXe siècle, comme une souveraineté territoriale absolue, même si elle n'a à peu près jamais été mise en pratique sous cette forme. Au XXIe siècle, elle est nécessairement quelque chose de partagé. Dans les Balkans, la conception du XIX ${ }^{\mathrm{e}}$ siècle, souvent professée par les leaders nationalistes, est synonyme d'insécurité et de nouveaux risques de guerre. Dans cette région comme dans le reste de l'Europe et du monde, une souveraineté allant de pair avec une sécurité réelle est forcément soumise à des conditions contenues dans des accords internationaux. En ce qui concerne le Kosovo, la souveraineté conditionnelle est le seul moyen de rendre l'indépendance acceptable pour ses voisins et d'accroître la sécurité ${ }^{7}$. La différence importante entre la souveraineté proposée ici pour le Kosovo et celle qui prévaut ailleurs réside en ceci que son caractère conditionnel n'est pas librement choisi par les intéressés et qu'elle devra être définie, codifiée et explicitement surveillée. En d'autres termes, l'indépendance conditionnelle a, dans le cas du Kosovo, un contenu spécifique. Elle signifie que l'indépendance ne pourra être acquise que sur la base de certaines conditions convenues et que, même après son obtention, la communauté internationale aura pour responsabilité de garantir que ces conditions restent réunies : en somme, la souveraineté sera explicitement surveillée.

\section{Vers un processus régional}

De quelle manière l'indépendance conditionnelle pourrait-elle se mettre en place ? Aussitôt que possible après les élections de novembre, une déclaration par laquelle les représentants de la communauté internationale se prononceraient clairement en faveur de cet objectif pourrait permettre d'éviter un débat stérile avec les dirigeants kosovars nouvellement élus. Même en l'absence de pareille déclaration, ces nouveaux dirigeants pourraient prendre des mesures qui s'inscriraient dans un processus susceptible de mener à l'indépendance :

- Exprimer leur engagement en faveur des conditions posées pour l'indépendance, en particulier la renonciation à la violence et l'inviolabilité des frontières.

- Lancer un processus de dialogue de grande ampleur, tant au Kosovo même qu'avec les pays voisins, sur les modalités de mise en œuvre de ces conditions. Il faudra y associer les dirigeants politiques, c'est-à-dire les représentants élus, et la société civile. Le dialogue avec la RFY revêtira une importance particulière car il permettra aux responsables comme aux simples citoyens de comprendre que le 
Kosovo ne fait plus partie de leur pays, mais désire coopérer sérieusement sur des sujets tels que la préservation des sites historiques ou le retour des réfugiés.

- Engager des négociations avec la MINUK sur un nouveau cadre constitutionnel transférant progressivement les attributions actuelles du Représentant spécial au gouvernement du Kosovo, à mesure que celui-ci construira son État et son administration.

Le dialogue doit s'inscrire dans un processus régional plus large, dans lequel la communauté internationale jouera un rôle de direction - non pas, toutefois, dans le sens où elle dicterait des solutions, mais parce qu'elle les rendrait possibles. Depuis quelques mois, on parle beaucoup d'une nouvelle conférence internationale qui réglerait définitivement les questions pendantes afin d'éliminer les risques de contagion qu'entraîne l'incertitude actuelle. Pourtant les conférences internationales n'ont pas manqué depuis dix ans - à Londres en 1992, à Dayton, à Rambouillet, pour ne mentionner que celles-là - qui soit n'ont pas abouti, soit n'ont pas su faire appliquer leurs décisions. Le problème de fond que pose ce type de réunions, où les décisions viennent d'en haut, est qu'elles passent à côté de la question centrale : le lien entre les décisions formelles (souveraineté, frontières, Constitutions) et la vie de tous les jours. La plupart des ex-Yougoslaves ont subi des régimes hautement centralisés, autoritaires, où tout était imposé d'en haut, ce qui explique en partie leur souci des questions formelles. Ce sont les frustrations de la vie quotidienne qui créent, à la base, les conditions du nationalisme. La question cruciale est de savoir comment décentraliser et démocratiser la vie quotidienne, car c'est alors que des questions comme la souveraineté ou les frontières perdent de leur importance. C'est dire que, si l'on ambitionne de parvenir à un règlement définitif, il faudra combiner des décisions à long terme sur le cadre formel et un rapport nouveau entre ce cadre formel et les processus informels; en d'autres termes, définir une souveraineté de type nouveau dans laquelle les symboles et les frontières compteront moins que par le passé. Pour être durable, ce règlement ne devra pas tomber du ciel comme ses prédécesseurs ; il faudra, par exemple, que les groupes civiques et les municipalités y soient davantage associés.

Ce type de processus régional, qui pourrait déboucher sur une conférence et un règlement définitif, est conforme aux propositions faites par le ministre yougoslave des Affaires étrangères Goran Svilanovic en faveur d'un « processus d'Helsinki » pour les Balkans. Il devra combiner trois composantes :

\footnotetext{
7. Ce même sentiment a été exprimé par le ministre albanais des Affaires étrangères Pascal Milo : « Nous voulons que ces frontières aient de moins en moins d'importance. Nous pensons rejoindre l'Union européenne, donc nous voulons que cette région soit une région de coopération. La philosophie du nationalisme et de l'extrémisme va à l'encontre des principes d'une Europe unie. Les nationalistes et les extrémistes veulent fermer les frontières et créer de petits États-nations. Pour nous, ce serait contre-productif ». Cité par Tim Judah, « Greater Albania », Survival 43 (2), 2001, p. 16.
} 
A. Les questions classiques du cadre d'Helsinki : frontières et droits des minorités.

B. Des questions nouvelles portant sur le rapport entre les décisions formelles (frontières, Constitutions, symboles de la souveraineté) et la vie quotidienne. Jusqu'à présent, ces aspects ont surtout été évoqués dans le cadre économique : ainsi, le Pacte de stabilité accorde une très grande attention à des mesures telles que la libéralisation du commerce ou la mise en place d'infrastructures communes. D'autres sujets n'ont pas moins d'importance, et le Conseil de coopération nordique pourrait servir ici de modèle. En voici quelques-uns :

- La réconciliation : le plus grand obstacle à un accord sur le statut futur de la province, à la cohabitation entre Serbes et Albanais au Kosovo ou entre Slaves et Albanais en Macédoine réside dans l'absence de vérité partagée, d'un récit communément admis - c'est-à-dire dépassant les versions mutuellement incompatibles existant actuellement - des événements de la décennie écoulée. Tous se sentent victimes, et leurs récits concurrents ainsi que leurs chronologies incohérentes font obstacle à une réconciliation régionale plus large. On a proposé, en Serbie comme en Bosnie, la création de commissions Vérité et Réconciliation. Mais si l'on n'y associe pas tous les peuples de la région, leurs travaux risquent d'aboutir, au mieux, à des vérités partielles, au pire à écrire une fois de plus des histoires distinctes pour chaque nation. C'est là qu'apparaît toute l'importance que pourrait revêtir un processus régional. Celui-ci pourrait également porter sur des mesures de confiance comme la recherche des disparus ou l'échange de prisonniers.

- Le patrimoine culturel : nous avons eu connaissance à Belgrade de la destruction du patrimoine culturel serbe du Kosovo au cours de la dernière année. En mai 2001, à Banja Luka, des manifestants se sont opposés à la reconstruction par des musulmans de mosquées détruites pendant la guerre. Cette région a un passé des plus riches, et les monuments des différentes nations et religions font partie d'un véritable patrimoine balkanique. Il faut donc discuter des moyens de protéger ce cadre culturel régional. L'engagement de chaque État à protéger le patrimoine culturel présent sur son territoire, en coopération avec ses voisins, constitue un test de maturité politique.

- La sécurité : une structure intégrée de sécurité couvrant toute la région et à laquelle la KFOR participerait au même titre que les forces locales pourrait permettre à la fois de professionnaliser ces forces et de prévenir les conflits. La coopération entre la KFOR et les forces serbes dans la vallée de Presevo pourrait ici servir de modèle.

Cette liste d'exemples n'est pas exhaustive ; d'autres sujets comme la santé publique, l'enseignement ou les médias pourraient y être ajoutés.

C. Des propositions d'institutionnalisation d'un cadre régional. Il ne manque vraiment pas d'initiatives régionales (le Pacte de stabilité, les accords de stabilisation et d'association avec l'Union européenne, les accords de partenariat avec l'OTAN) 
ni de formes d'engagement de la communauté internationale (la MINUK, le Bureau du Haut Représentant en Bosnie, la présence de l'OTAN en Albanie et en Macédoine, par exemple). Ce qui manque, c'est la clarté des choix et la constance de l'engagement politique. Par exemple, le Pacte de stabilité lancé en fanfare à Sarajevo à l'été 1999 avait suscité de grands espoirs, mais l'élan politique est ensuite retombé. Il importe certainement de réfléchir aux moyens de rationaliser ces différentes initiatives en les intégrant à un cadre balkanique unique, lié à l'Union européenne, et qui aurait son siège dans la région, peut-être à Sarajevo.

Si un processus régional de ce type doit nécessairement être autorisé au plus haut niveau, à la fois par les gouvernements de la région et par la communauté internationale, il doit aussi offrir le cadre d'un processus allant de bas en haut et impliquant des débats à tous les niveaux de la société. À leur modeste échelle, les négociations de la vallée de Presevo, là aussi, donnent l'exemple.

\section{Conclusion}

Notre rapport estimait que, dans certaines circonstances exceptionnelles équivalant à une catastrophe humanitaire imminente ou en cours, il pouvait y avoir lieu de recourir à une force internationale en se passant éventuellement de l'autorité des Nations unies. Mais il est alors essentiel que le recours à la force se conforme rigoureusement à une série de directives constituant un cadre de principes appelés à régir tous les cas d'intervention humanitaire (le texte de ces principes figure aux pp. 193 à 195 du rapport). Le rapport affirmait d'ailleurs que, même dans le cas d'une intervention humanitaire sanctionnée par l'autorité des Nations unies, la communauté internationale a le devoir de se conformer à ces directives. La Commission estime que l'expérience du Kosovo depuis 1999 confirme la validité de ces positions. Afin que ces directives, ou d'autres légèrement différentes, acquièrent une plus grande portée, il serait hautement souhaitable qu'elles soient adoptés formellement par les organes des Nations unies, en particulier par le Conseil de sécurité et l'Assemblée générale. On pourrait charger la Commission du droit international d'en proposer une rédaction faisant autorité.

L'expérience des mois écoulés depuis la publication du rapport a également démontré le bien-fondé (aussi bien que les insuffisances) de ce cadre en ce qui concerne les devoirs qui incombent aux acteurs internationaux après l'intervention. Il en est ainsi des principes 9 et 11. Le principe 9 est formulé comme suit : « Il conviendra de se conformer beaucoup plus strictement au droit de la guerre et au droit international humanitaire que dans le cadre d'opérations militaires ordinaires. Cela vaut pour tous les aspects de l'opération militaire, y compris toute occupation se prolongeant après le cessez-le-feu ». La Commission estime que la KFOR mérite tous les éloges pour s'y être constamment pliée jusqu'à ce jour. 
Cependant, la KFOR et la MINUK n'ont pas fait preuve d'une diligence suffisante pour protéger les minorités menacées, en particulier au cours des premières phases de l'occupation, et cela a rendu possible ce qu'on a souvent appelé l'« épuration ethnique à l'envers », avec de nombreux cas de mauvais traitements infligés à la minorité serbe du Kosovo et à ses alliés, en particulier les Rom. Des efforts plus importants auraient dû être déployés dès le début de l'occupation afin de protéger les civils et d'établir la primauté du droit. Il aurait également fallu davantage se préoccuper de garantir la sécurité des frontières et d'empêcher l'approvisionnement de groupes armés dans la zone de sécurité terrestre, dans le sud de la Serbie et en Macédoine. La deuxième phrase du principe 9 devrait donc être remaniée comme suit pour tenir compte de cet impératif : «Cela vaut pour tous les aspects de l'opération militaire, y compris toute occupation se prolongeant au-delà du cessez-le-feu, et impose en particulier aux forces d'occupation et à leurs homologues administratifs le devoir d'accorder la plus grande priorité à la protection des composantes menacées de la population civile, y compris la prévention des actes de vengeance et de représailles $\gg$. S'il ne fait pas de doute que la légitimité de l'intervention de l'OTAN a souffert de ce que ce devoir n'ait pas été rempli, ce n'est toutefois pas au point que la Commission doive revenir sur son soutien à ce qui a été accompli car, tout bien considéré, c'est bien cette action qui a mis fin à un désastre humanitaire qui ne cessait de s'aggraver.

De manière générale, la Commission estime que l'évolution récente des Balkans confirme l'importance de ses propositions initiales. Loin de fournir un motif de reporter à plus tard une solution durable du conflit du Kosovo, la chute de Milosevic offre l'occasion d'un dialogue véritable entre le Kosovo et la Serbie. Mais, à l'évidence, la communauté internationale, en particulier les puissances occidentales qui ont soutenu l'intervention et portent donc une plus grande part de responsabilité, doivent exercer une pression sur les partenaires pour qu'ils saisissent cette occasion. Le risque existe bel et bien que les gouvernements occidentaux soient en train de répéter les erreurs historiques commises à l'égard du Kosovo au début des années quatre-vingt-dix : en n'accordant pas leur soutien au mouvement de résistance non violente à l'oppression serbe et en excluant des négociations de Dayton la question du Kosovo, ils avaient laissé le conflit s'envenimer et, qui plus est, avaient donné à entendre aux Kosovars que le monde extérieur ne prêtait attention qu'à la violence - ce qui donna naissance à l'UCK.

En effet, c'est peut-être une situation analogue qui se représente aujourd'hui. Le Kosovo aurait dû, sitôt après la chute de Milosevic, bénéficier de la même priorité de la part des gouvernements occidentaux que l'arrestation de ce dernier. Les arguments utilisés contre son transfèrement à La Haye sont exactement les mêmes que ceux que certains invoquent pour ne pas régler dès à présent la question du statut du Kosovo. Or l'arrestation de Milosevic s'est révélée positive pour la démocratisation de la Serbie. Une décision sur l'avenir du Kosovo pourrait très 
bien avoir, elle aussi, des effets positifs. À l'inverse, le report des décisions a d'ores et déjà des conséquences négatives. L'incertitude nourrit la violence, tant au Kosovo que dans les États voisins. De plus, les responsables politiques kosovars ne se voient guère incités à tenter de faire ce que la communauté internationale n'a pas su faire : établir un climat de sécurité à l'intérieur comme à l'extérieur.

Les élections de novembre 2001 au Kosovo offrent une nouvelle occasion d'avancer la proposition d'indépendance conditionnelle. La communauté internationale pourrait prévenir de futurs conflits en présentant une proposition d'indépendance à terme. Les responsables politiques kosovars, pour leur part, pourraient tirer parti de l'état de grâce consécutif aux élections pour commencer à mettre en œuvre les conditions dont cette proposition serait assortie.

La Commission continue de considérer l'intervention de l'OTAN comme légitime. Cependant, si l'on néglige de s'engager suffisamment en faveur de l'avenir du Kosovo et de la région et de prendre les initiatives politiques (assorties du soutien militaire et économique approprié) indispensables à une solution durable, cette légitimité pourrait en souffrir. Nous estimons donc qu'il convient de s'engager en faveur d'une indépendance conditionnelle pour le Kosovo et d'un processus de dialogue et de coopération pour la région. Tous ceux qui ont pris part à l'intervention de l'OTAN partagent une responsabilité : celle de préserver la légitimité de l'opération par un suivi constructif.

Traduit de l'anglais par Rachel Bouyssou

Liste des membres de la Commission

\section{Président}

Richard Goldstone, Afrique du Sud

\section{Membres}

Grâce d'Almeida, Bénin*

Hanan Ashrawi, Palestine*

Akiko Domoto, Japon

Richard Falk, États-Unis

Oleg Grinevsky, Russie*

Michael Ignatieff, Canada

\section{Co-président \\ Carl Tham, Suède}

Mary Kaldor, Royaume-Uni

Martha Minow, États-Unis

Jacques Rupnik, France

Theo Sommer, Allemagne

Jan Urban, République tchèque

\footnotetext{
* Grâce d'Almeida, Hanan Ashrawi et Oleg Grinevsky n'ont pas pu participer à l'ensemble des travaux et ne sont donc pas associés au texte du rapport.
} 